\section{Kidney \\ Blood Pressure Research}

\title{
Polymorphisms in the SLC12A3 Gene En- coding Sodium-Chloride Cotransporter are Associated with Hypertension: A Family-Based Study in the Mongolian Population
}

\author{
Caiyan An ${ }^{a}$ Junqing Liang ${ }^{a}$ Kejin Zhang ${ }^{b}$ Xiulan Su
}

${ }^{a}$ Clinical Research Center of the Affiliated Hospital, Inner Mongolia Medical University, Hohhot, ${ }^{b}$ Key Laboratory of Resource Biology and Biotechnology in Western China (Ministry of Education), College of Life Science, Institute of Population and Health, Northwest University, Xi'an, China

\section{Key Words}

Hypertension • SLC12A3 • Family-based association test (FBAT) • Mongolian • Haplotype

\begin{abstract}
Background/Aims: Hypertension or persistent high blood pressure (BP) is a leading cause of death worldwide. Extensive evidence indicates that the thiazide-sensitive $\mathrm{Na}^{+}-\mathrm{Cl}^{-}$cotransporter (NCC) affects BP via regulation of renal sodium reabsorption. However, the relationship between genetic variants of the NCC-encoding SLC12A3 gene and hypertension in the Mongolian population is still ambiguous. In this study, we aimed to genotype an extended cohort of hypertensive Mongolian families for polymorphisms in the SLC12A3 locus. Methods: Eighty-eight families with a history of hypertension, including parents, offspring, and relatives underwent clinical testing. Family-based association tests and haplotype analysis were used to evaluate the association between hypertension and polymorphisms in the SLC12A3 locus. Results: We identified three single nucleotide polymorphisms (SNPS), one in the SLC12A3 coding region $(p=0.05)$ and two in the intron $(p=0.02$ and $p=0.07)$, which were significantly associated with the hypertension phenotype. Haplotype-specific association tests confirmed the correlation of these SNPs with hypertension $(p<0.05)$. Conclusion: These results suggest that SNPs in the SLC12A3 gene confer susceptibility to hypertension in the Mongolian population. Further research is needed to validate the functional role of SLC12A3 polymorphisms in hypertension.
\end{abstract}




\section{Kidney Blood Pressure Research}

Kidney Blood Press Res 2016;41:18-28

DOI: 10.1159/000368543

Published online: January 08, 2016

(C) 2016 The Author(s). Published by S. Karger AG, Base

www.karger.com/kbr

An et al.: SLC12A3 is Associated with Hypertension in Mongolians

Introduction

Hypertension or persistent high blood pressure (BP) is a condition that significantly increases the risk of heart and cerebrovascular diseases, thus posing a severe threat to human health. Worldwide, more than one billion people have hypertension [1], which is the leading cause of mortality, accounting for approximately 7.5 million deaths each year [2-4]. Essential or primary hypertension, defined as high BP for which there are no secondary causes such as renovascular disease and renal failure, is the most common form, accounting for $90 \%-95 \%$ of all cases; secondary hypertension due to kidney, artery, heart, or endocrine system disorders covers the remaining 5\%-10\% [5]. Previous genome-wide association studies [6, 7] have identified genetic loci associated with hypertension, suggesting that it results from a complex interaction of genetic mechanisms and environmental factors. However, although a number of genetic polymorphisms and rare genetic variants associated with slight or significant effects on BP and hypertension have been identified $[8,9]$, the genetic basis of hypertension is still poorly understood.

It is established that salt homeostasis maintained by the kidneys is directly associated with BP and that renal sodium reabsorption plays an important role in the development of hypertension. The up-regulation of renal sodium reabsorption triggers BP elevation via the concomitant increase in water reabsorption to maintain plasma sodium concentration at or near $140 \mathrm{mM}$; the resultant increase in intravascular volume augments venous blood return to the heart, thereby raising cardiac output and directly leading to hypertension [10]. The thiazide-sensitive $\mathrm{Na}^{+}-\mathrm{Cl}^{-}$cotransporter (NCC) in the kidney transports sodium and chloride ions from the surrounding fluid into the cells of nephron distal convoluted tubule, and accumulating evidence [11] indicates that genetic variation of the NCC-encoding SLC12A3 gene is associated with kidney sodium reabsorption and hypertension via effects on NCC functional activity. For example, SLC12A3 mutation, Arg919Cys in exon 24, increase NCC activity, resulting in the up-regulation of kidney sodium reabsorption [11]. In addition to this, a recent study on pendrin and NCC dKO mice concluded that pendrin and NCC are predominantly active during salt depletion (and or in response to aldosterone) [12]. Association studies have shown that SLC12A3 mutation (Arg904Gln and G2736A) demonstrated a significant relationship with the risk of hypertension $[13,14]$. Chang et al. [15] have reported positive association of tag single nuclear polymorphism (SNP) rs7204044 with hypertension in both the Mongolian and Han populations in China. Genetic variants of the SLC12A3 gene have also been shown to affect the response to BP-reducing drugs such as thiazide diuretics [16]. However, other studies have not found correlation between SLC12A3 polymorphisms and hypertension. Zhang et al. [17] have reported the lack of association between Arg904Gln substitution and essential hypertension based on a meta-analysis, while other studies did not observe the relationship of reported SLC12A3 polymorphisms with hypertension in rats [18] and humans [19]. Similarly, the Arg904Gln polymorphism in NCC did not show correlation with hypertension, as evidenced by the investigation of two ethnic groups in Northwest China [20] and our earlier study in the Uyghurs, Kazaks, and Mongolians [21], ethnic minorities in China. However, these and other related studies based on random or specific populations have not performed stratification for genetic admixture which may be a confounding factor affecting the conclusion about the link between $S L C 12 A 3$ genetic variants and hypertension. In addition, the results have not been validated in replication studies.

In our previous study, we have investigated the relationship between the SLC12A3 gene and hypertension and found that two tag SNPs are predisposing genetic factors for hypertension in the Han population dominant in China [21]. In the current study, we aimed to analyze the association of the common SLC12A3 variants with the occurrence of essential hypertension among the inhabitants of the Inner Mongolia region, which has not been yet established. To address this, we used a family-based association test (FBAT) to study a cohort of hypertensive ethnic Mongolians with extended pedigree. 


\section{Kidney Blood Pressure Research}

An et al.: SLC12A3 is Associated with Hypertension in Mongolians

\section{Materials and Methods}

\section{Subjects}

The investigation of a family-based cohort of hypertensive and normotensive individuals was performed at two separate locations of Xilingol League in the Inner Mongolia Autonomous Region of China, the Xianghuang county, and the city of Xilinhot. Subjects with essential hypertension were identified based on systolic BP (SBP) $\geq 140 \mathrm{mmHg}$ and/or diastolic BP (DBP) $\geq 90 \mathrm{mmHg}$, or by the use of at least one antihypertensive drug, and were included in the study as probands. The relatives of the probands were tracked and diagnosed based on the same criteria using the same procedure; normotensive relatives were also recruited. Probands with secondary hypertension, severe cardiovascular disease, kidney disease and diabetes mellitus, as well as their relatives were excluded. In total, 424 individuals (236 hypertensive patients and their relatives; among them, 211 women and 213 men) comprising 88 extended pedigrees were tracked, recruited, and investigated. All participants were ethnic Mongolians. The study was conducted in accordance with the ethical standards established by the United States National Institute of Health and was approved by the Ethical Committee of Affiliated Hospital of Inner Mongolia Medical University. Written informed consent was obtained from all participants or their parents/guardians.

\section{Subjects' evaluation}

Participants' characteristics, including age, gender, ethnicity, and family structure, were recorded. Patients were in sitting position when blood pressure was measured as described previously [21]: 1) three times with 2-min intervals between the measurements; 2) SBP and DPB were recorded to the nearest 2 $\mathrm{mmHg}$ at the appearance of the first (phase I) and disappearance of the fifth (phase V) Korotkoff sounds, respectively; 3 ) three consecutive measurements were averaged. Body mass index (BMI) was calculated as the body weight in kilograms divided by the square of height in meters. Such clinical parameters as total plasma cholesterol (TC), triglycerides (TG), high-density lipoprotein cholesterol (HDL-C), low-density lipoprotein cholesterol (LDL-C), and fasting blood glucose were measured after 8-h overnight fasting at a local hospital using routine methods. Indirect clinical diagnosis was also made by physicians for incapacitated, deceased, or absent family members based on the information offered by their guardians or close relatives.

\section{Genotyping}

In this study, SLC12A3 was considered as a candidate gene for the evaluation of genetic polymorphism effects on hypertension. SNPs were selected from the dbSNP (http://www.ncbi.nlm.nih.gov/projects/SNP/) and the International HapMap Project (http://hapmap.ncbi.nlm.nih.gov/) datasets using the HaploView 3.2 software package [22] by the method of Gabriel et al. [23]. Briefly, the strategy and criteria for tag SNP selection were as follows: a) location in the target gene or 1,000-bp flanking regions, b) preference for potentially functional SNPs, c) minor allele frequency (MAF) $>10 \%$ in the Asian population (Han Chinese in Beijing + Japanese in Tokyo); and d) $r^{2}$ threshold > 0.80. Finally, 15 SNPs in the SLC12A3 gene were selected (Table 1); they were located within $3.4 \mathrm{~kb}$, one SNP was mapped $2.0 \mathrm{~kb}$ upstream of the SLC12A3 gene, one synonymous codon was mapped to the exon region, and the rest were located in the intron region.

Genomic DNA was obtained from $1 \mathrm{~mL}$ venous blood using the AxyPrep-96 DNA Extraction Kit (Axygen, Union City, CA, USA) and subjected to multiplex PCR-ligase detection. Then, $1 \mu \mathrm{L}$ of the amplified product was mixed with $1 \mu \mathrm{L}$ of GS-500 ROX fluorescent molecular weight standards (Applied Biosystems, Foster City, CA, USA) and $1 \mu \mathrm{L}$ deionized formamide, denatured at $95^{\circ} \mathrm{C}$ for $2 \mathrm{~min}$, and resolved by electrophoresis in $5 \%$ polyacrylamide gels containing $5 \mathrm{~mol} / \mathrm{L}$ ureophil. Images were captured using the GENESCANTM 672 software and analyzed with GeneMapper 3.0. Strict quality control measures were implemented during genotyping, with more than $99 \%$ concordance in duplicate randomly selected samples.

\section{Statistical analysis}

The demographic information and baseline data of hypertensive and normotensive family members were assessed by compare means test (Independent - Sample T Test) and exact Chi-square test using the SPSS13.0 software (SPSS Inc., Chicago, IL, USA). In order to reduce influence of relationship, only parents (166 female and 148 male) were included and analyzed for demographic information and baseline comparison. Prior to the analysis, family samples were tested with the PEDCHECK program [24] to identify Mende- 


\section{Kidney Blood Pressure Research}

An et al.: SLC12A3 is Associated with Hypertension in Mongolians

Table 1. The location of selected tag SNPs and Hardy-Weinberg equilibrium test results

\begin{tabular}{lcclllll}
\hline SNPs & Position ${ }^{a}$ & $\begin{array}{c}\text { Distance } \\
(\mathrm{kb}) \mathrm{b}\end{array}$ & Location & ObsHET c & HWp ${ }^{\mathrm{d}}$ & MAFe & Alleles \\
\hline rs4784733 & $16: 56865094$ & - & Upstream & 0.359 & 1.0000 & 0.231 & C:T \\
rs2304478 & $16: 56865628$ & 0.53 & Intron & 0.226 & 0.9086 & 0.133 & G:A \\
rs13306673 & $16: 56867019$ & 1.39 & Intron & 0.189 & 0.8189 & 0.099 & T:C \\
rs2289119 & $16: 56878991$ & 11.97 & Intron & 0.347 & 0.1190 & 0.272 & G:A \\
rs8043560 & $16: 56883033$ & 4.04 & Intron & 0.389 & 0.8321 & 0.251 & C:T \\
rs2304483 & $16: 56884041$ & 1.01 & Intron & 0.409 & 0.2380 & 0.345 & G:A \\
rs5803 & $16: 56887057$ & 3.02 & Coding exon & 0.369 & 1.0000 & 0.246 & T:C \\
rs7187932 & $16: 56897792$ & 10.74 & Intron & 0.418 & 0.3198 & 0.260 & G:A \\
rs6499858 & $16: 56901203$ & 3.41 & Intron & 0.523 & 0.2846 & 0.400 & T:C \\
rs11644728 & $16: 56905634$ & 4.43 & Intron & 0.226 & 0.5920 & 0.138 & G:C \\
rs8049280 & $16: 56906216$ & 0.58 & Intron & 0.267 & 0.5946 & 0.169 & C:T \\
rs7204044 & $16: 56908797$ & 2.58 & Intron & 0.221 & 0.4835 & 0.115 & G:A \\
rs2010501 & $16: 56911627$ & 2.83 & Intron & 0.405 & 0.6189 & 0.305 & C:T \\
rs2399594 & $16: 56912285$ & 0.66 & Intron & 0.421 & 0.6763 & 0.323 & A:G \\
rs711746 & $16: 56912892$ & 0.61 & Intron & 0.451 & 0.2574 & 0.451 & A:G \\
\hline
\end{tabular}

aPhysical position of SNPs in the GRCh38 data set. ${ }^{\mathbf{b}}$ Distance from the adjacent marker. ${ }^{\text {ObsHET, observed }}$ heterozygosity. dHWp, p values of the Hardy-Weinberg equilibrium test. eMAF, minimum allele frequency.

lian inconsistencies in the pedigree data. Family-based association analyses were performed using the FBAT Program 1.5.5 (http://www.biostat.harvard.edu/ fbat/default.html) which represents a unified approach to testing valid associations between marker alleles and traits applicable to any phenotype, sampling structure, and pattern of missing marker alleles $[25,26]$; similar to the transmission/disequilibrium test (TDT), FBAT is immune to confounding by admixture. With missing parents, FBAT is similar to, but more powerful than, the reconstruction-combined TDT and considerably more powerful than the sib TDT [27]. If Mendelian inconsistencies were detected, pedigree sub-families were not considered useful for association analyses, although the FBAT approach is completely robust against model misspecification. Individual marker genotypes were tested for the Hardy-Weinberg equilibrium and analyzed for linkage disequilibrium (LD) by Haploview [22]. We first tested each marker separately for excessive allele transmission under difference genetic models (e.g. additive, dominant and recessive models). As Horvath et al. [28] suggested, the results under additive model were prior to consider, since the true genetic model was unknown. Then we analyzed excessive haplotype transmission for the consistency with the markers identified by single-site analysis. Haplotypes were reconstructed using the HBAT command in FBAT, which utilizes the expectation-maximization algorithm for SNPs with obvious overtransmission $(p<0.10)$. The statistical power of this investigation with the present sample size was estimated by the Quanto 1.2.4. program for power and sample size calculations in genetic studies [29]. Statistical significance was considered at $p<0.05$. To control the risk of false discoveries, a so-called $q$-value which estimates the proportion of false discoveries among all significant markers [30] was calculated for each $p$-value used as the threshold for declaring significance.

In silico analysis was performed to evaluate the potential biological significance of SNPs related to the following: 1) transcription factor binding site or promoter region; 2) splice site alteration; and 3) expression of a novel protein with different structure-functional properties. The TFSEARCH program (http:// www.gene-regulation.com/links_tools.html) was used to search transcription factor binding sites identified in vertebrates [31] using the 85.0 threshold score within 50-bp sequence around each SNP. The Neural Network Promoter Prediction, Promoter 2.0 Prediction, and McPromoter MM:II tools were used to detect promoter sites generated or lost because of alternative SNP alleles. Splice site estimation was performed with the MasEnNCCan software based on the maximum entropy principle [32] and the splice site predictor at the Berkeley Drosophila Genome Project based on neural networks [33]. Furthermore, 300-bp SNP flanking regions were analyzed by the ORF Finder (http://www.ncbi.nlm.nih.gov/gorf/gorf.html) and Conserved Domain Database (http://www.ncbi.nlm.nih.gov/Structure/cdd/wrpsb.cgi) to identify possible ORFs, 


\section{Kidney Blood Pressure Research}

conserved domains, and related protein families. The signature-recognition tools Phobius (http://www.ebi. ac.uk/Tools/pfa/phobius/) and InterProScan (http://www.ebi.ac.uk/interpro/) were used to evaluate the effects of SNPs on the structural features of polypeptides.

\section{Results}

Demographic and baseline characteristics of the participants

The present study included 424 participants with familial hypertension covering 88 extended pedigrees of more than 100 nuclear families; for each family, one to nine patients were identified. Participants' demographic and baseline data are presented in Table 2. For 314 individuals, the data on BP, gender, age, and five basic clinical parameters were available. Information on the heritage and pedigree genetic structure of the remaining 110 participants could be obtained and these individuals were also included in the FBAT analysis, although the related basic variables were missing. Normotensive and hypertensive subjects showed significant differences in gender, age, SBP, and DBP (Table 2). More men had hypertensive status than women $(p=0.002)$, and normal BP was more characteristic of younger people $(p$ $<0.001$ ). Hypertensive subjects had significantly higher SBP (mean $=154.29 \mathrm{mmHg}, 95 \% \mathrm{CI}$ [151.03-157.55]) and DBP (mean $=100.84 \mathrm{mmHg}$, 95\%CI [99.10-102.58]) than normotensive subjects (SBP, mean $=117.16 \mathrm{mmHg}$, 95\%CI [115.32-119.00] and DBP, mean $=75.80$ $\mathrm{mmHg}, 95 \% \mathrm{CI}[74.60-77.00])(p<0.001)$. There was a statistically significant difference in two clinical variables, TG and LDL-C, which were higher in hypertensive participants $(p=$ 0.031 and $p=0.049$, respectively).

Genotyping profile and LD structure of SLC12A3 SNPS

The identified 15 SNPs did not show significant deviation from Hardy-Weinberg distribution (Table 1). Heterozygosity ranged from $18.9 \%$ to $52.3 \%$ and most MAFs were more than $10 \%$, except for the rs 13306673 site (MAF $=9.9 \%)$. Figure 1 shows the LD structure created by HaploView, which identified three blocks of tag SNPs with higher LD, consistent with the rough distribution in the HapMap data set.

Table 2. Demographic and clinical characteristics of study participants

\begin{tabular}{|c|c|c|c|}
\hline Characteristics & $\begin{array}{l}\text { Normotensive } \\
\text { group [95\% CI] } \\
(\mathrm{n}=135)\end{array}$ & $\begin{array}{l}\text { Hypertensive } \\
\text { group [95\% CI] } \\
(\mathrm{n}=179)\end{array}$ & $p$ value \\
\hline $\begin{array}{l}\text { Median age, } \\
\text { years (range) }\end{array}$ & $40(15-82)$ & $53(15-87)$ & $<0.001^{\mathrm{b}}$ \\
\hline Gender, female/male & $85 / 50$ & $81 / 98$ & $0.002^{b}$ \\
\hline SBP, mmHga & $117.16[115.32,119.00]$ & $154.29[151.03,157.55]$ & $<0.001^{b}$ \\
\hline DBP, mmHga & $75.80[74.60,77.00]$ & $100.84[99.10,102.58]$ & $<0.001^{b}$ \\
\hline BMI, $\mathrm{kg} / \mathrm{m}^{2}$ & $25.17[24.37,25.98]$ & $25.00[24.21,25.80]$ & 0.430 \\
\hline $\mathrm{TC}, \mathrm{mM}$ & $4.42[4.21,4.63]$ & $4.79[4.54,5.05]$ & 0.106 \\
\hline TG, mM & $1.26[1.07,1.45]$ & $1.71[1.36,2.06]$ & $0.031^{b}$ \\
\hline HDL-C, mmol/L & $1.39[1.33,1.44]$ & $1.33[1.27,1.40]$ & 0.134 \\
\hline LDL-C, mmol/L & $2.89[2.70,3.04]$ & $3.14[2.96,3.31]$ & $0.049 \mathrm{~b}$ \\
\hline $\mathrm{FBG}, \mathrm{mmol} / \mathrm{L}$ & $6.39[6.22,6.57]$ & $6.32[6.01,6.63]$ & 0.699 \\
\hline
\end{tabular}




\section{Kidney Blood Pressure Research}

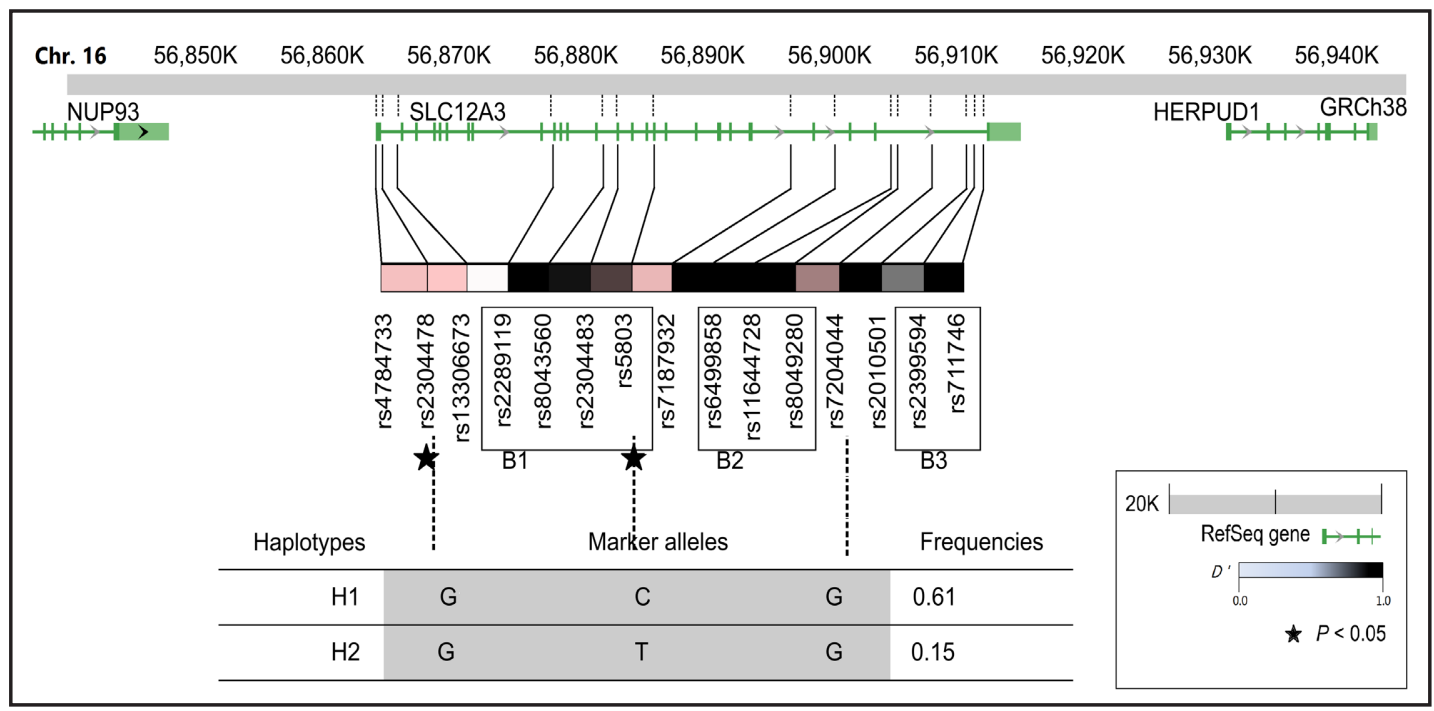

Fig. 1. Markers used in the association analyses. Symbols and positions of the markers relative to the SLC12A3 gene and adjacent genes are shown. Haplotype frequencies for three unfavorable SNPs with MAFs of $\geq 5 \%$ are indicated. Fifteen SNPs are grouped into three blocks (B1, B2, and B3) using the method of Gabriel et al. with the $r^{2}>0.8$ threshold [23]. SLC12A3, solute carrier family 12 (sodium/chloride transporter), member 3; UNP93, nuclear pore complex protein Nup93; HERPUD1, ubiquitin-like domain member 1; GRCh38, new human genome assembly (GenBank, December 2013).

\section{Genotype and allele distributions of SLC12A3 SNPS}

Single marker association analysis identified two SNPs significantly associated with hypertension in our family cohort in both additive biallelic and multi-allelic models. As shown in Table 3, the G allele of rs2304478 showed significant positive correlation with hypertension $(z=2.36, p=0.02$ in additive model and dominant model), while the T allele of rs5803 transmitted in families with one or more hypertensive members showed significant negative correlation with hypertension $(z=-1.95, p=0.05$ in additive model, $p=0.01$ in dominant model). The same results were obtained for rs7204044, where the $\mathrm{G}$ allele also showed an obvious trend for transmission in hypertensive patients $(z=1.81, p=0.07)$ under additive model. However, the data for three SNPs did not withstand correction for multiple testing.

\section{Haplotype analysis}

Structure analysis of haplotypes containing three SNPs and showing obvious overtransmission in hypertensive patients is presented in Table 4. The HBAT test indicated that three haplotypes with two-window size showed significant association with hypertension $(p=$ 0.012 for $\mathrm{rs} 2304478(\mathrm{G}) / \mathrm{rs} 5803(\mathrm{C}), p=0.016$ for $\mathrm{rs} 5803(\mathrm{C}) / \mathrm{rs} 7204044(\mathrm{G})$, and $p=0.006$ for rs2304478(G)/rs7204044(G)). Furthermore, the haplotype $G / C / G$ containing three SNPs was also transmitted among hypertensive patients showing positive association $(z=$ $2.65, p=0.008$ ). All $p$ values withstood correction for multiple testing and demonstrated statistically significant association between the haplotypes and hypertension (all $p<0.05$ ) (Table 4 and Fig. 1).

\section{In silico functional analysis of SNPS}

We next performed detailed in silico analysis of SNPs that showed significant association with hypertension as single markers, including rs2304478, rs5803, and rs7204044. No transcription factor binding sites or promoters were affected by these SNPs. rs5803 was likely to be located within a splice site determined by MasEnNCCan based on the maximum entropy principle (the maximum entropy model score -1.38 for the $\mathrm{T}$ allele and -9.13 for the $\mathrm{C}$ allele). The analysis with ORF Finder showed that rs2304478 (a 300-bp sequence) might encode a 


\section{Kidney Blood Pressure Research}

59-amino acid peptide having 94\% identity with solute carrier family 12 member 3 isoform $\mathrm{X} 1$ of Chrysemys picta bellii, whereas a putative peptide comprising the rs5803 site had 91\% identity with Homo sapiens $\mathrm{Na}^{+}-\mathrm{Cl}^{-}$ electro neutral NCC (46 amino acids) containing the $2 \mathrm{a} 30$ domain of a $\mathrm{K}^{+}$$\mathrm{Cl}^{-}$cotransporter. No match was found for a putative rs7204044-encoded peptide. However, the rs2304478 and rs5803 alternative alleles did not show obvious effects on the putative encoded peptides.

Statistical power analys is of genetic testing

Statistical power analysis by QUANTO 1.2.4 indicated that the present study based on more than 100 trio families (in which both parents and at least one child were affected by hypertension) had $>80 \%$ power in the FBAT test (MAF $>0.25, \mathrm{OR}>2.0, \mathrm{k}_{\mathrm{p}}=0.20$ ), which guarantees the reliability and accuracy of our analysis results.

Table 3. Family-based association test for tag SNPs under additive and dominant genetic models

\begin{tabular}{lllllll}
\hline \multirow{2}{*}{ Marker } & \multirow{2}{*}{ Alleles $^{\mathrm{a}}$} & Allele frequency & \multicolumn{2}{l}{ Test statistics } & \multicolumn{2}{l}{$p$-FBAT } \\
& & & $z^{\mathrm{b}}$ & CHISQ $^{\mathrm{c}}$ & Additive & Dominant \\
\hline rs4784733 & C:T & $0.22 / 0.78$ & 1.36 & 1.84 & 0.17 & 0.41 \\
rs2304478 & G:A & $0.86 / 0.14$ & 2.36 & 5.56 & $0.022^{\mathrm{d}}$ & $0.02^{\mathrm{d}}$ \\
rs13306673 & T:C & $0.10 / 0.90$ & 0.69 & 0.48 & 0.49 & 0.75 \\
rs2289119 & G:A & $0.73 / 0.27$ & 1.42 & 2.02 & 0.16 & 0.07 \\
rs8043560 & C:T & $0.25 / 0.75$ & -0.41 & 0.17 & 0.68 & 0.46 \\
rs2304483 & G:A & $0.65 / 0.35$ & 1.24 & 1.54 & 0.22 & 0.22 \\
rs5803 & T:C & $0.26 / 0.74$ & -1.95 & 3.80 & $0.05 \mathrm{~d}$ & $0.01 \mathrm{~d}$ \\
rs7187932 & G:A & $0.74 / 0.26$ & -0.43 & 0.18 & 0.67 & 0.07 \\
rs6499858 & T:C & $0.38 / 0.62$ & 1.07 & 1.34 & 0.28 & 0.37 \\
rs11644728 & G:C & $0.88 / 0.12$ & 0.06 & 0.01 & 0.95 & 0.86 \\
rs8049280 & C:T & $0.16 / 0.84$ & -0.56 & 0.32 & 0.57 & 0.49 \\
rs7204044 & G:A & $0.89 / 0.11$ & 1.81 & 3.26 & 0.07 & 0.16 \\
rs2010501 & C:T & $0.28 / 0.72$ & -0.77 & 0.59 & 0.44 & 0.13 \\
rs2399594 & A:G & $0.69 / 0.31$ & -0.67 & 0.45 & 0.50 & 0.27 \\
rs711746 & A:G & $0.56 / 0.44$ & -0.83 & 0.70 & 0.41 & 0.45 \\
\hline
\end{tabular}

aAlleles detected in more than 10 informative families. bz-score calculated based on a biallelic marker model; positive $z$ values and $p<0.05$ indicate a high-risk haplotype. ${ }^{\mathrm{C}} \mathrm{CHISQ}$ a statistic parameter calculated based on a multiple marker model. dSignificant $p$ values $(<0.05)$ are in bold.

Table 4. Haplotype-specific analysis of identified SNPs associated with hypertension

\begin{tabular}{|c|c|c|c|c|c|}
\hline Haplotypes & Frequencya & $\operatorname{Var}(\mathrm{S})^{\mathrm{b}}$ & $\begin{array}{c}\text { Test statistics } \\
(z)\end{array}$ & $p$-HBATc & Global $p$ d \\
\hline \multicolumn{5}{|l|}{ rs2304478/ rs5803 } & 0.042 e \\
\hline $\mathrm{G} / \mathrm{C}$ & 0.70 & 8.98 & 2.50 & $0.012 \mathrm{e}$ & \\
\hline $\mathrm{G} / \mathrm{T}$ & 0.16 & 5.87 & -1.03 & 0.302 & \\
\hline \multicolumn{5}{|l|}{ rs5803/ rs7204044 } & $0.046 \mathrm{e}$ \\
\hline $\mathrm{C} / \mathrm{G}$ & 0.69 & 8.45 & 2.41 & $0.016^{\mathrm{e}}$ & \\
\hline $\mathrm{T} / \mathrm{G}$ & 0.22 & 6.22 & -1.34 & 0.181 & \\
\hline \multicolumn{5}{|c|}{ rs2304478/rs7204044 } & $0.022 \mathrm{e}$ \\
\hline $\mathrm{G} / \mathrm{G}$ & 0.76 & 9.63 & 2.72 & $0.006 \mathrm{e}^{\mathrm{C}}$ & \\
\hline $\mathrm{A} / \mathrm{G}$ & 0.15 & 6.50 & -1.63 & 0.102 & \\
\hline \multicolumn{5}{|c|}{ rs2304478/ rs5803/ rs7204044 } & 0.027 e \\
\hline $\mathrm{G} / \mathrm{C} / \mathrm{G}$ & 0.61 & 9.89 & 2.65 & $0.008 \mathrm{e}$ & \\
\hline $\mathrm{G} / \mathrm{T} / \mathrm{G}$ & 0.15 & 5.08 & -0.44 & 0.657 & \\
\hline
\end{tabular}

aHaplotypes with higher frequencies (>0.10) in more than 10 informative families. ${ }^{b}$ Estimated variance for general statistics compared with a null model. ${ }^{c} p$ values of the HBAT test. d $p$ values of the asymptotic global HBAT test for all haplotypes with more than 0.10 frequencies within one-window size. eSignificant $p$ values $(<0.05)$ are in bold. 


\section{Kidney Blood Pressure Research}

Kidney Blood Press Res 2016;41:18-28

DOI: 10.1159/000368543

Published online: January 08, 2016

(C) 2016 The Author(s). Published by S. Karger AG, Base

www.karger.com/kbr

An et al.: SLC12A3 is Associated with Hypertension in Mongolians

Discussion

Hypertension results from complex interactions of genetic traits and environmental factors, where genetic background could account for 30\%-50\% of BP variations in the general population [34]. The Mongolians, one of the minor ethnic groups in China, have a high prevalence of hypertension: the crude hypertension rate amounts to $50 \%$ among elderly inhabitants (age > 55 years) of a pastoral area of the Inner Mongolia Autonomous Region. Moreover, there is a tendency for familial aggregation [35], suggesting a critical role of genetic factors in the high prevalence of hypertension among the Mongolians. In the present study, we focused on the effects of genetic variation in the SLC12A3 gene on hypertension in the Mongolian population in China. To the best of our knowledge, this is the first study to investigate the effect of SLC12A3 genetic variants on hypertension in a family-based cohort. The association we observed was particularly evident in an extended family cohort which allowed elimination or effective reduction of the influence of environmental factors and is immune to confounding by admixture [36].

Genetic variation, including SNPs, plays an important role in the regulation of gene expression via mRNA splicing, degradation, and translation, and may affect protein structure and biological function [37]. Recent efforts in exploring molecular mechanisms of hypertension have revealed polymorphisms in the genes encoding renal sodium and potassium transporters, which contribute to hypertension susceptibility in the general population [38]. The variations in the SLC12A3 gene, including common and rare SNPs and other mutations, affect the regulation of solute transport and sodium reabsorption in the kidneys and show a close relationship with hypertension and Gitelman's syndrome characterized by low BP $[11,13,39]$. In the present study, two SNPs (rs2304478 and rs5803) in the SLC12A3 gene showed significant association with hypertension in the Mongolian population; one of the alleles was transmitted in the family and positively (rs2304478) or negatively (rs5803) correlated with hypertension. rs7204044 was also included in our analysis based on previous findings in which it is positively associated with hypertension [15], although in this study, it showed statistically insignificant positive association with hypertension. The results of haplotype analysis were consistent with those of single-site analysis in that haplotypes containing pedigree-transmitted SNP alleles showed significant association with hypertension, both for window sizes of two and three SNP haplotypes by the HBAT method. No significant association with hypertension was detected for other selected SNPs in the current study on the Mongolians, as well as in previous studies on other populations [15, 20, 21].

The biological functions of the three identified SNPs and the influence of the underlying regulatory genetic mechanisms on clinical outcome for hypertensive patients remain to be elucidated. The in silico analysis which included SNP 150-bp flanking regions indicated that rs5803, a synonymous SNP, may be involved in the alternative splicing of the SLC12A3 mRNA of electroneutral NCC harboring the $2 \mathrm{a} 30$ domain of the $\mathrm{K}^{+}-\mathrm{Cl}^{-}$cotransporter, which regulates a number of physiological processes, including BP [40]. A part of the putative rs2304478-containing peptide (17 amino acids) showed high identity with solute carrier family 12 member 3 isoform X1 from C. picta bellii. Thus, we cannot rule out a possibility that rs5803 and rs2304478 can be biologically meaningful SNPs, although their functional significance has not been determined. SLC12A3-encoded NCC involved in $\mathrm{Na}^{+}-\mathrm{Cl}^{-}$reabsorption in the kidney is important for electrolyte homeostasis and is a potential target for treating high BP. Therefore, SLC12A3 genetic polymorphisms can affect renal electrolyte reabsorption via regulation of NCC functional activity, and are likely to be associated with BP control and hypertension. Our present investigation showing the correlation between SNPs in the SLC12A3 gene and hypertension confirms this notion.

Our study has several limitations. First, in this association analysis of complex traits, the possibility of spurious correlations must be considered. Two approaches utilized in the current study might have reduced the likelihood of false-positive findings: a family-based cohort, which excludes confounding due to population admixture, and validation of stati- 


\section{Kidney Blood Pressure Research}

stically significant association using multimarker haplotype and global tests, which ensures that true association is revealed.

Second, the analyzed sample size was relatively modest. Several SNPs, including rs7204044, which did not achieve nominal statistical significance, had low MAFs $(<15 \%)$, and it is possible that some of these SNPs may demonstrate correlation with hypertension in a larger population. Thus, despite a possibility of a type II error, the association we revealed can still be considered robust.

Third, as in most association studies, it is unclear which, if any, of the tested SNPs may be causally related to the development of hypertension phenotype, although certain functional evaluation was performed in silico. Among the examined SNPs, only one (rs5803) is located in the SLC12A3 coding sequence. This SNP which represents a synonymous change in the Ala714 site was found to be associated with hypertension in this study, possibly because of its potential effect on alternative splicing [41]. However, no functional significance had been reported for rs2304478 and rs7204044 at the time of our investigation (September 2014), suggesting the need for further research to identify causal associations.

Fourth, it was difficult to determine the contribution of individual markers and their inter-relationship because the three SNPs were not located in a single region with strong LD; therefore, the influence of each SNP on hypertension was independent of others or still unknown. Modest sample size allowed only the analysis of direct associations and precluded that of indirect associations occurring through LD and conditioning markers; thus, cumulative effects of unfavorable alleles could not be estimated.

Fifth, the effect of clinical variables may still have an impact on our conclusions. Despite sample control, significant differences were still found between hypertensive and normotensive participants (gender, age, TG, and LDL-C); therefore, further stratification and adjustment are required.

Sixth, functional gain-of-function SNPs in SLC12A3 gene would be expected to be associated with hyperkalemia, metabolic acidosis and hypercalciuria (the opposite of Gitelman and in the same direction as seen in Gordon syndromes). Nevertheless, these parameters were not measured at that time of our collecting samples. It is a limitation for the present study and these parameters will be tested in further association study. Finally, the $p$ values determined by single-marker analysis did not withstand correction for multiple comparisons, and the possibility of a type I error cannot be excluded.

\section{Conclusion}

In summary, we observed an association between the SLC12A3 gene and hypertension in a family-based cohort from the population of Inner Mongolia. Further studies are needed to clarify the effect of specific SLC12A3 polymorphisms on BP and risk for hypertension, which would provide scientific basis for the development of novel therapeutic approaches to treat hypertension using NCC-mediated signaling pathways as drug targets.

\section{Disclosure Statement}

The authors declare no conflicts of interest.

\section{Acknowledgments}

We are grateful to all participants in this study. This work was supported by a grant from the National Natural Science Foundation of China (\#81260058). 


\section{Kidney \\ Blood Pressure Research}

An et al.: SLC12A3 is Associated with Hypertension in Mongolians

\section{References}

1 Lawes CM, Vander Hoorn S, Rodgers A: Global burden of blood-pressure-related disease. Lancet 2008;371:1513-1518.

2 Danaei G, Ding EL, Mozaffarian D, Taylor B, Rehm J, Murray CJ, Ezzati M: The preventable causes of death in the united states: Comparative risk assessment of dietary, lifestyle, and metabolic risk factors. PLoS Med 2009;6:e1000058.

3 Lopez AD, Mathers CD, Ezzati M, Jamison DT, Murray CJ: Global and regional burden of disease and risk factors, 2001: Systematic analysis of population health data. Lancet 2006;367:1747-1757.

4 Ezzati M, Lopez AD, Rodgers A, Vander Hoorn S, Murray CJ: Selected major risk factors and global and regional burden of disease. Lancet 2002;360:1347-1360.

5 Carretero OA, Oparil S: Essential hypertension. Part i: Definition and etiology. Circulation 2000;101:329335.

6 International Consortium for Blood Pressure Genome-Wide Association Studies, Ehret GB, Munroe PB, Rice KM, Bochud M, Johnson AD, Chasman DI, Smith AV, Tobin MD, Verwoert GC, Hwang SJ, Pihur V, Vollenweider P, O’Reilly PF, Amin N, Bragg-Gresham JL, Teumer A, Glazer NL, Launer L, Zhao JH, Aulchenko Y, Heath S, Sõber S, Parsa A, Luan J, Arora P, Dehghan A, Zhang F, Lucas G, Hicks AA, Jackson AU, Peden JF, Tanaka T, Wild SH, Rudan I, Igl W, Milaneschi Y, Parker AN, Fava C, et al.: Genetic variants in novel pathways influence blood pressure and cardiovascular disease risk. Nature 2011;478:103-109.

7 Org E, Eyheramendy S, Juhanson P, Gieger C, Lichtner P, Klopp N, Veldre G, Doring A, Viigimaa M, Sober S, Tomberg K, Eckstein G, Kelgo P, Rebane T, Shaw-Hawkins S, Howard P, Onipinla A, Dobson RJ, Newhouse SJ, Brown M, Dominiczak A, Connell J, Samani N, Farrall M, Caulfield MJ, Munroe PB, Illig T, Wichmann HE, Meitinger T, Laan M: Genome-wide scan identifies cdh13 as a novel susceptibility locus contributing to blood pressure determination in two european populations. Hum Mol Genet 2009;18:2288-2296.

8 He J, Kelly TN, Zhao Q, Li H, Huang J, Wang L, Jaquish CE, Sung YJ, Shimmin LC, Lu F, Mu J, Hu D, Ji X, Shen C, Guo D, Ma J, Wang R, Shen J, Li S, Chen J, Mei H, Chen CS, Chen S, Li J, Cao J, Lu X, Wu X, Rice TK, Gu CC, Schwander K, Hamm LL, Liu D, Rao DC, Hixson JE, Gu D: Genome-wide association study identifies 8 novel loci associated with blood pressure responses to interventions in han chinese. Circ Cardiovasc Genet 2013;6:598-607.

9 Kato N, Takeuchi F, Tabara Y, Kelly TN, Go MJ, Sim X, Tay WT, Chen CH, Zhang Y, Yamamoto K, Katsuya T, Yokota M, Kim YJ, Ong RT, Nabika T, Gu D, Chang LC, Kokubo Y, Huang W, Ohnaka K, Yamori Y, Nakashima E, Jaquish CE, Lee JY, Seielstad M, Isono M, Hixson JE, Chen YT, Miki T, Zhou X, Sugiyama T, Jeon JP, Liu JJ, Takayanagi R, Kim SS, Aung T, Sung YJ, Zhang X, Wong TY, Han BG, Kobayashi S, Ogihara T, Zhu D, Iwai N, Wu JY, Teo YY, Tai ES, Cho YS, He J: Meta-analysis of genome-wide association studies identifies common variants associated with blood pressure variation in east asians. Nat Genet 2011;43:531-538.

10 Lifton RP, Gharavi AG, Geller DS: Molecular mechanisms of human hypertension. Cell 2001;104:545-556.

11 Keszei AP, Tisler A, Backx PH, Andrulis IL, Bull SB, Logan AG: Molecular variants of the thiazide-sensitive $\mathrm{Na}+\mathrm{Cl}$ - cotransporter in hypertensive families. J Hypertens 2007;25:2074-2081.

$12 \mathrm{Xu}$ J, Barone S, Brooks MB, Soleimani M: Double knockout of carbonic anhydrase II (caii) and na(+)-cl(-) cotransporter (ncc) causes salt wasting and volume depletion. Cell Physiol Biochem 2013;32:173-183.

13 Melander O, Orho-Melander M, Bengtsson K, Lindblad U, Rastam L, Groop L, Hulthen UL: Genetic variants of thiazide-sensitive nacl-cotransporter in gitelman's syndrome and primary hypertension. Hypertension 2000;36:389-394.

14 Matsuo A, Katsuya T, Ishikawa K, Sugimoto K, Iwashima Y, Yamamoto K, Ohishi M, Rakugi H, Ogihara T: G2736a polymorphism of thiazide-sensitive na-cl cotransporter gene predisposes to hypertension in young women. J Hypertens 2004;22:2123-2127.

15 Chang PY, Zhao LG, Su XL: Association of tsc gene variants and hypertension in mongolian and han populations. Genet Mol Res 2011;10:902-909.

16 Matayoshi T, Kamide K, Takiuchi S, Yoshii M, Miwa Y, Takami Y, Tanaka C, Banno M, Horio T, Nakamura S, Nakahama H, Yoshihara F, Inenaga T, Miyata T, Kawano Y: The thiazide-sensitive na(+)-cl(-) cotransporter gene, $\mathrm{c} 1784 \mathrm{t}$, and adrenergic receptor-beta3 gene, $\mathrm{t727c}$, may be gene polymorphisms susceptible to the antihypertensive effect of thiazide diuretics. Hypertens Res 2004;27:821-833.

17 Zhang F, Yang Y, Hu D, Lei H, Wang Y: Lack of an association between tsc gene arg904gln polymorphisms and essential hypertension risk based on a meta-analysis. Genet Mol Res 2012;11:3511-3517. 


\section{Kidney \\ Blood Pressure Research}

18 Song Y, Herrera VL, Filigheddu F, Troffa C, Lopez LV, Glorioso N, Ruiz-Opazo N: Non-association of the thiazide-sensitive na,cl-cotransporter gene with polygenic hypertension in both rats and humans. J Hypertens 2001;19:1547-1551.

19 Aoi N, Nakayama T, Sato N, Kosuge K, Haketa A, Sato M, Soma M: Case-control study of the role of the gitelman's syndrome gene in essential hypertension. Endocr J 2008;55:305-310.

20 Wang XF, Lin RY, Wang SZ, Zhang LP, Qian J, Lu DR, Wen H, Jin L: Association study of variants in two ion-channel genes (tsc and clcnkb) and hypertension in two ethnic groups in northwest china. Clin Chim Acta 2008;388:95-98.

21 Chang PY, Zhang XG, Su XL: Lack of association of variants of the renal salt reabsorption-related genes slc12a3 and clc-kb and hypertension in mongolian and han populations in inner mongolia. Genet Mol Res 2011;10:948-954.

22 Barrett JC, Fry B, Maller J, Daly MJ: Haploview: Analysis and visualization of ld and haplotype maps. Bioinformatics 2005;21:263-265.

23 Gabriel SB, Schaffner SF, Nguyen H, Moore JM, Roy J, Blumenstiel B, Higgins J, DeFelice M, Lochner A, Faggart M, Liu-Cordero SN, Rotimi C, Adeyemo A, Cooper R, Ward R, Lander ES, Daly MJ, Altshuler D: The structure of haplotype blocks in the human genome. Science 2002;296:2225-2229.

24 O'Connell JR, Weeks DE: Pedcheck: A program for identification of genotype incompatibilities in linkage analysis. Am J Hum Genet 1998;63:259-266.

25 Laird NM, Horvath S, Xu X: Implementing a unified approach to family-based tests of association. Genet Epidemiol 2000;19:S36-42.

26 Rabinowitz D, Laird N: A unified approach to adjusting association tests for population admixture with arbitrary pedigree structure and arbitrary missing marker information. Hum Hered 2000;50:211-223.

27 Horvath S, Xu X, Laird NM: The family based association test method: Strategies for studying general genotype--phenotype associations. Eur J Hum Genet 2001;9:301-306.

28 Horvath S, Laird NM: A discordant-sibship test for disequilibrium and linkage: No need for parental data. Am J Hum Genet 1998;63:1886-1897.

29 Gauderman W, Morrison J: Quanto 1.1: A computer program for power and sample size calculations for genetic-epidemiology studies. http://hydra.Usc.Edu/gxe.

30 Storey JD, Tibshirani R: Statistical significance for genomewide studies. Proc Natl Acad Sci USA 2003;100:9440-9445.

31 Heinemeyer T, Wingender E, Reuter I, Hermjakob H, Kel AE, Kel OV, Ignatieva EV, Ananko EA, Podkolodnaya OA, Kolpakov FA, Podkolodny NL, Kolchanov NA: Databases on transcriptional regulation: TRANSFAC, TRRD and COMPEL. Nucleic Acids Res 1998;26:362-367.

32 Yeo G, Burge CB: Maximum entropy modeling of short sequence motifs with applications to RNA splicing signals. J Comput Biol 2004;11:377-394.

33 Reese MG, Eeckman FH, Kulp D, Haussler D: Improved splice site detection in genie. J Comput Biol 1997;4:311-323.

34 Coffman TM: Under pressure: The search for the essential mechanisms of hypertension. Nat Med 2011;17:1402-1409.

35 Gong P, Zhang H, Chi W, Ge W, Zhang K, Zheng A, Gao X, Zhang F: An association study on the polymorphisms of dopaminergic genes with working memory in a healthy chinese han population. Cell Mol Neurobiol 2012;32:1011-1019.

36 Laird N: Family-based association test (fbat); in els, john wiley \& sons, ltd 2011;DOI: 10.1002/9780470015902.a0022500.

37 Shastry BS: Snps: Impact on gene function and phenotype. Methods Mol Biol 2009;578:3-22.

38 Welling PA: Rare mutations in renal sodium and potassium transporter genes exhibit impaired transport function. Curr Opin Nephrol Hypertens 2014;23:1-8.

39 Ji W, Foo JN, O'Roak BJ, Zhao H, Larson MG, Simon DB, Newton-Cheh C, State MW, Levy D, Lifton RP: Rare independent mutations in renal salt handling genes contribute to blood pressure variation. Nat Genet 2008;40:592-599.

40 Arroyo JP, Kahle KT, Gamba G: The slc12 family of electroneutral cation-coupled chloride cotransporters. Mol Aspects Med 2013;34:288-298.

41 Caceres JF, Kornblihtt AR: Alternative splicing: Multiple control mechanisms and involvement in human disease. Trends Genet 2002;18:186-193. 